\title{
Improvement of Omega-3-rich Microalgae Biomass Production to Support Qatar Food
}

\section{Security}

Maroua Cherif, Touria Bounnit, Hareb Al Jabri and Imen Saadaoui

Algal Technologies Program, Center for Sustainable Development, Qatar University, Doha- Qatar

\section{ABSTRACT}

\begin{abstract}
Microalgae are considered as one of the most promising solution based on its ability to produce the essential elements needed for food and feed such as lipids, carbohydrates, proteins... In the Centre for Sustainable Development, Qatar University, a local and diverse Culture Collection of Cyanobacteria and Microalgae was established and maintained. Among them, QUCCCM50, Tetraselmis sp. isolate was selected as the most promising local isolate for feed supplement production due to its high nutritional potential, fast growth and spontaneous harvesting. The aim of the current project consists on optimizing all physicochemical condition leading to improving the production of lipid production mainly polyunsaturated fatty acids (PUFAs) importantly, Omega-3. For that purpose, 3 different strategies were tested. The growth rate, metabolites production and FAME profiling were assessed during the algae growth.

The results obtained showed that the best condition for lipid accumulation was $100 \%$ salinity showing $25 \%$ increase in lipid content. The same culture condition led to an increase in the omega 3 content notably the ALA fatty acid comparing to the control growth conditions.
\end{abstract}

\section{OBJECTIVES}

Study the ability of a local microalgae isolate to grow under different growth conditions.

Optimizing the growth conditions for the marin isolate leading to high lipid and FAME content key characteristic for supporting Qatar food security

\section{LITERATURE REVIEW}

The worldwide continuous increasing of population led to an increasing awareness about the food security. Microalgae emerged as alternative and have been the subject 0 applied research for their commercial and industrial potential.. They have the ability to transform inorganic matters into high valuable organic components such as, carbohydrates lipid and protein $(63-92 \%$ of the dry weight (Becker et al., 1986) suitable for different applications e.g. fuel, feed, pharmaceuticals etc.

Moreover, their biochemical composition car be modified by altering their growth condition such as temperatures, salinity and nutrients to improve their quality and hence can be used as food additive to increase the nutritional value (Valente et al., 2006).

Most commonly used strains are Chlorella sp, Tetraselmis sp, Spirullina sp... etc.

\section{METHODOLOGY}
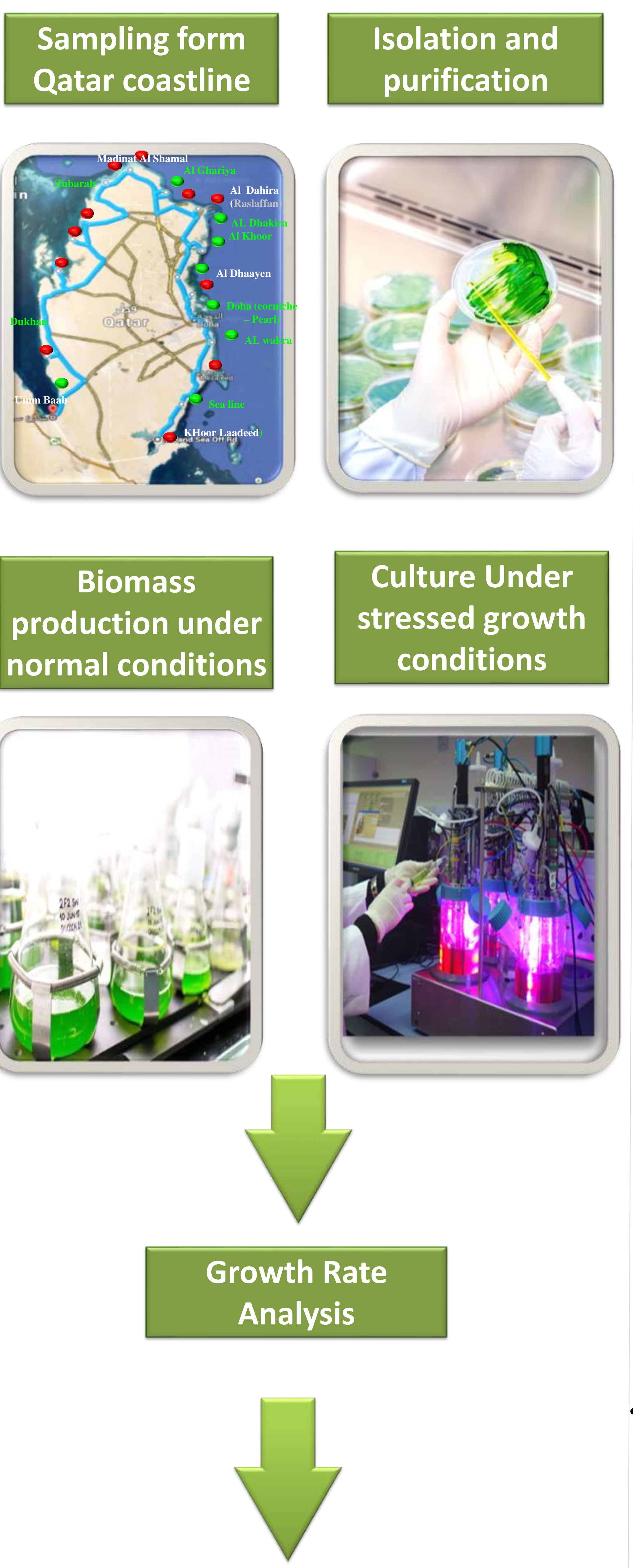

lipid extraction (A) and Characterization (B)
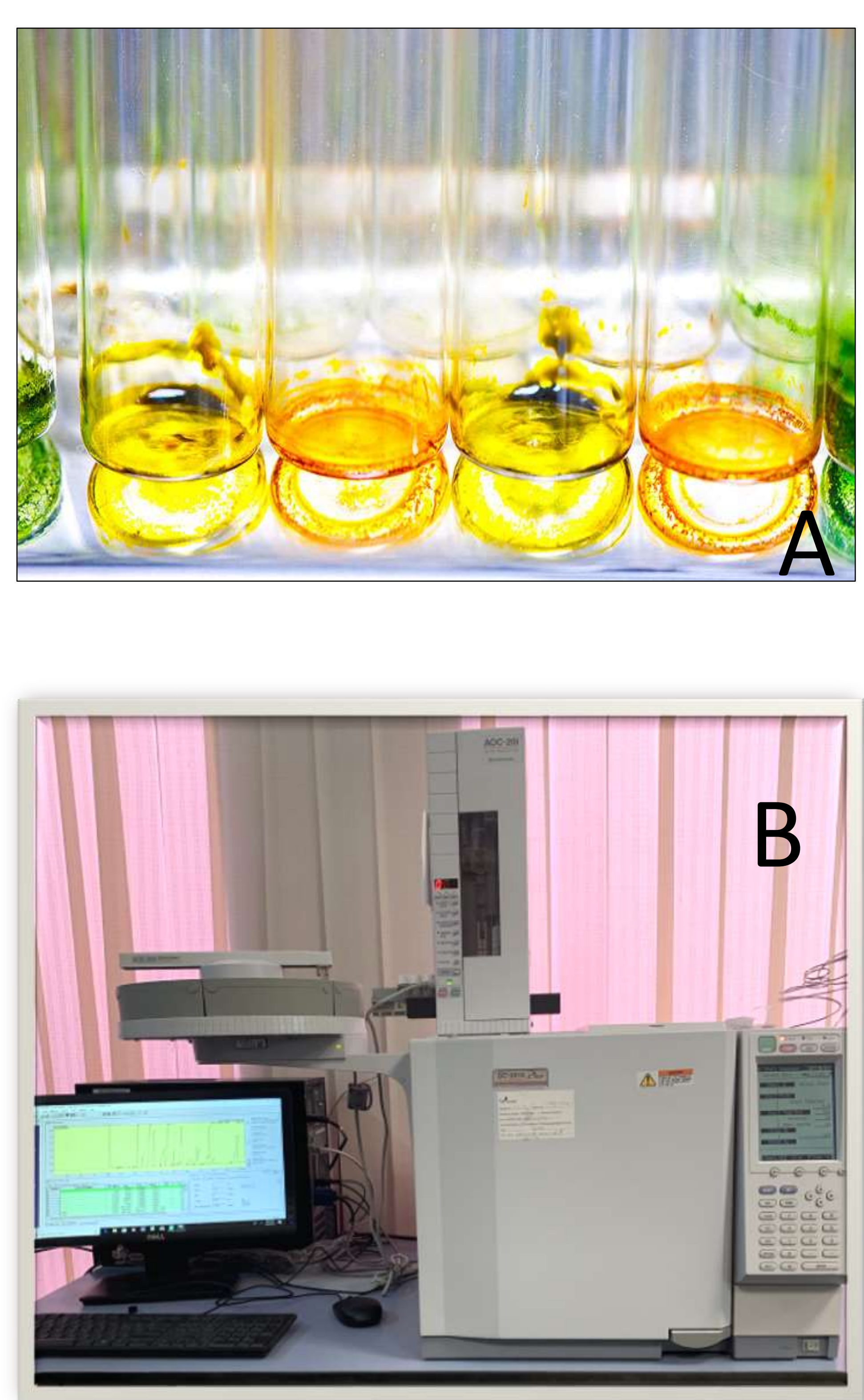

RESULTS \& DISCUSSION

1. Growth rate and biomass productivity Analysis
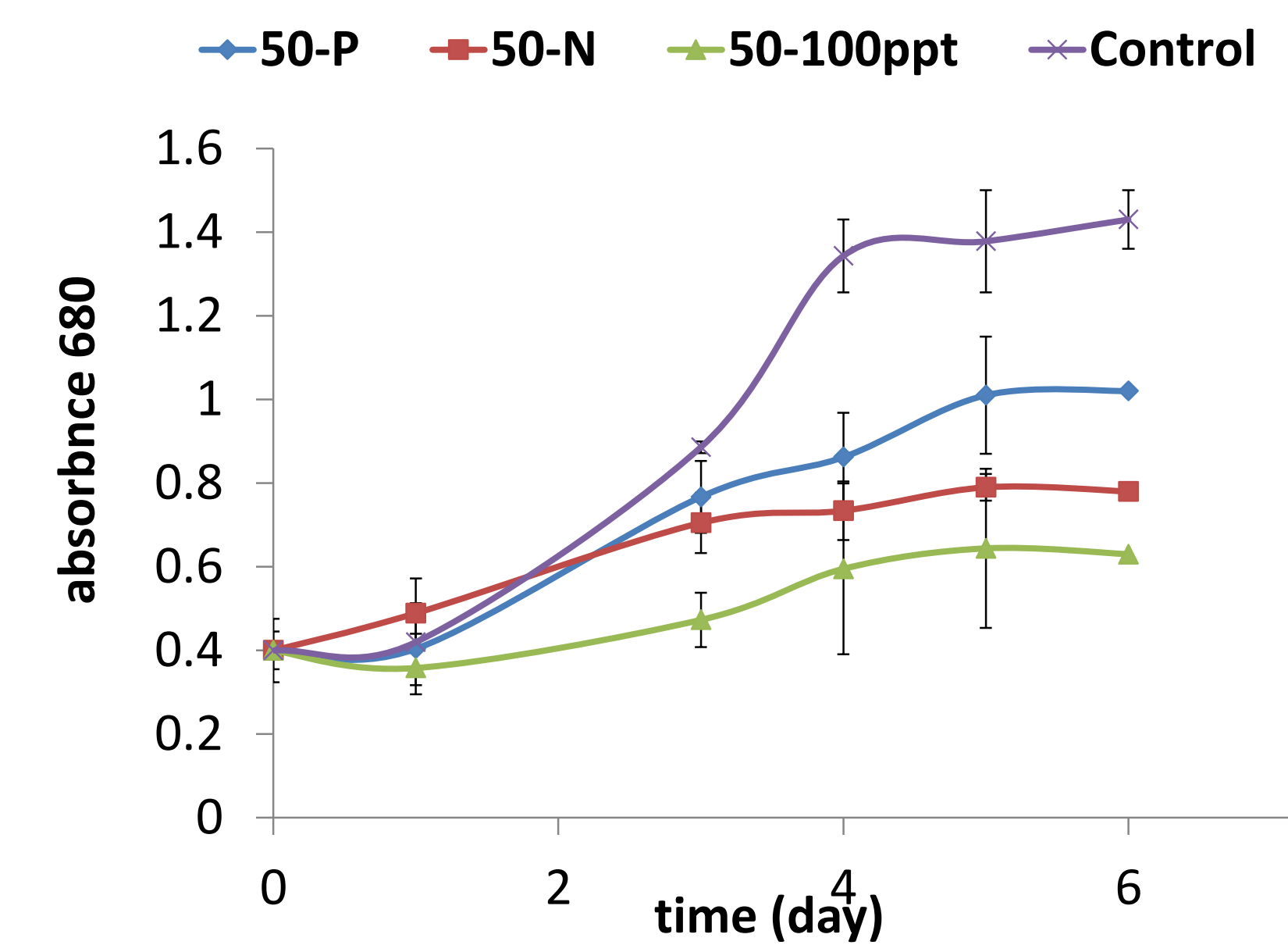

Figure.1. The growth rate analysis of the -QUCCCM50 under the different cultivation regimes: 50-P: Cultivation under P-starvation; 50-P: Cultivation under N-starvation; 50-100ppt: Cultivation under 100ppt salinity.

The results show that comparing to the control, all the stress conditions hindered the growth of the algae. The highest inhibition was observed at 100ppt salinity.

Table 1 . Growth rate and biomass productivity under different culture conditions

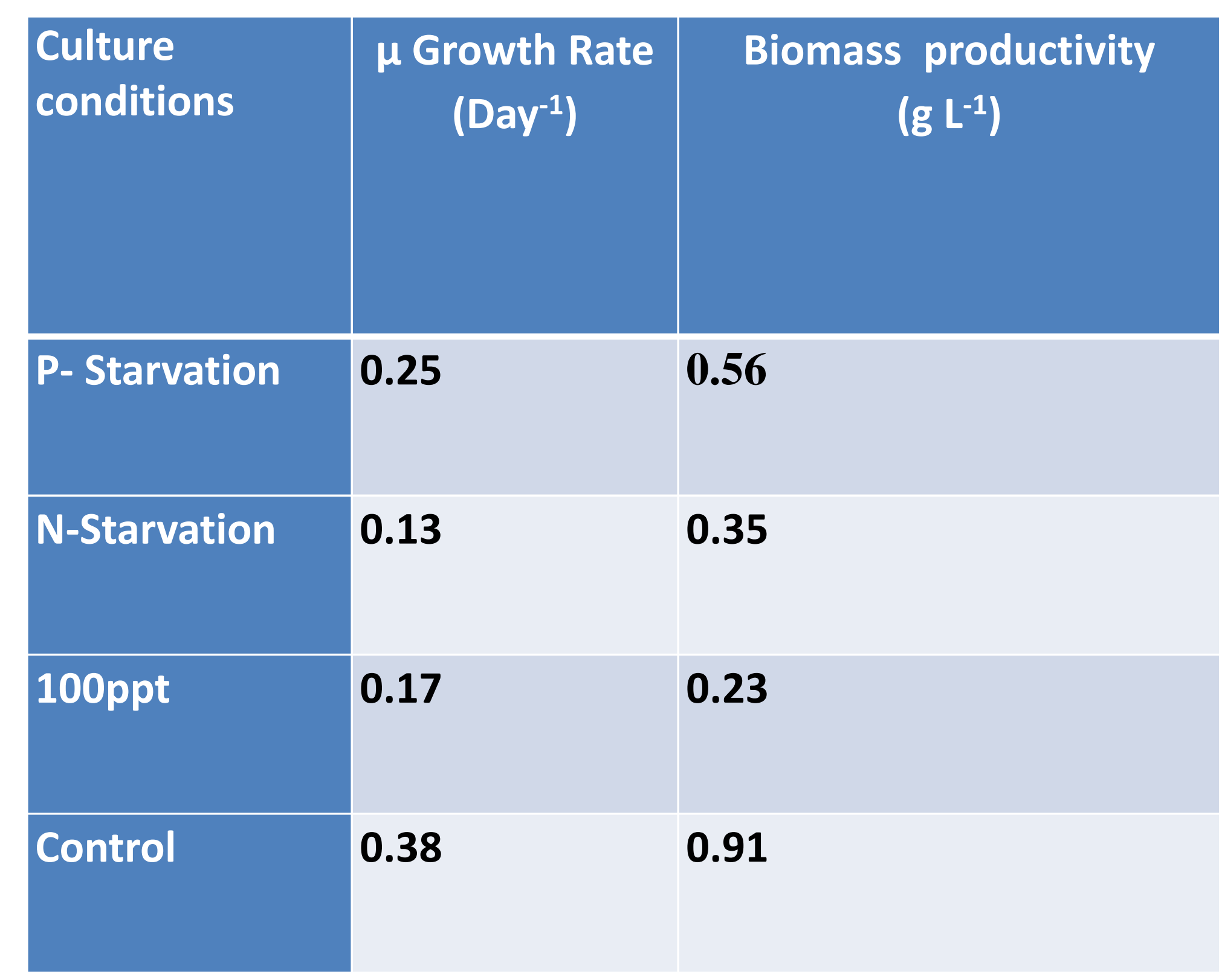

The comparison of the QUCCCM50 growth under the different cultivation regimes with the control showed that all stress conditions affect the growth rate and the biomass production differently (figure1, 2). Indeed, QUCCCM50 showed a $\mathrm{u}$ of $\mathbf{0 . 3}$ day $\mathbf{- 1}$ under control condition. However, a decrease of with $22-50 \%$ in terms of $u$ and biomass productivity was observed in the case of all stress conditions studied. Our results confirmed that the stress conditions altered the algae growth.

\section{Chlorophyll analysis:}

Table 2 . Chlorophyll content under different culture

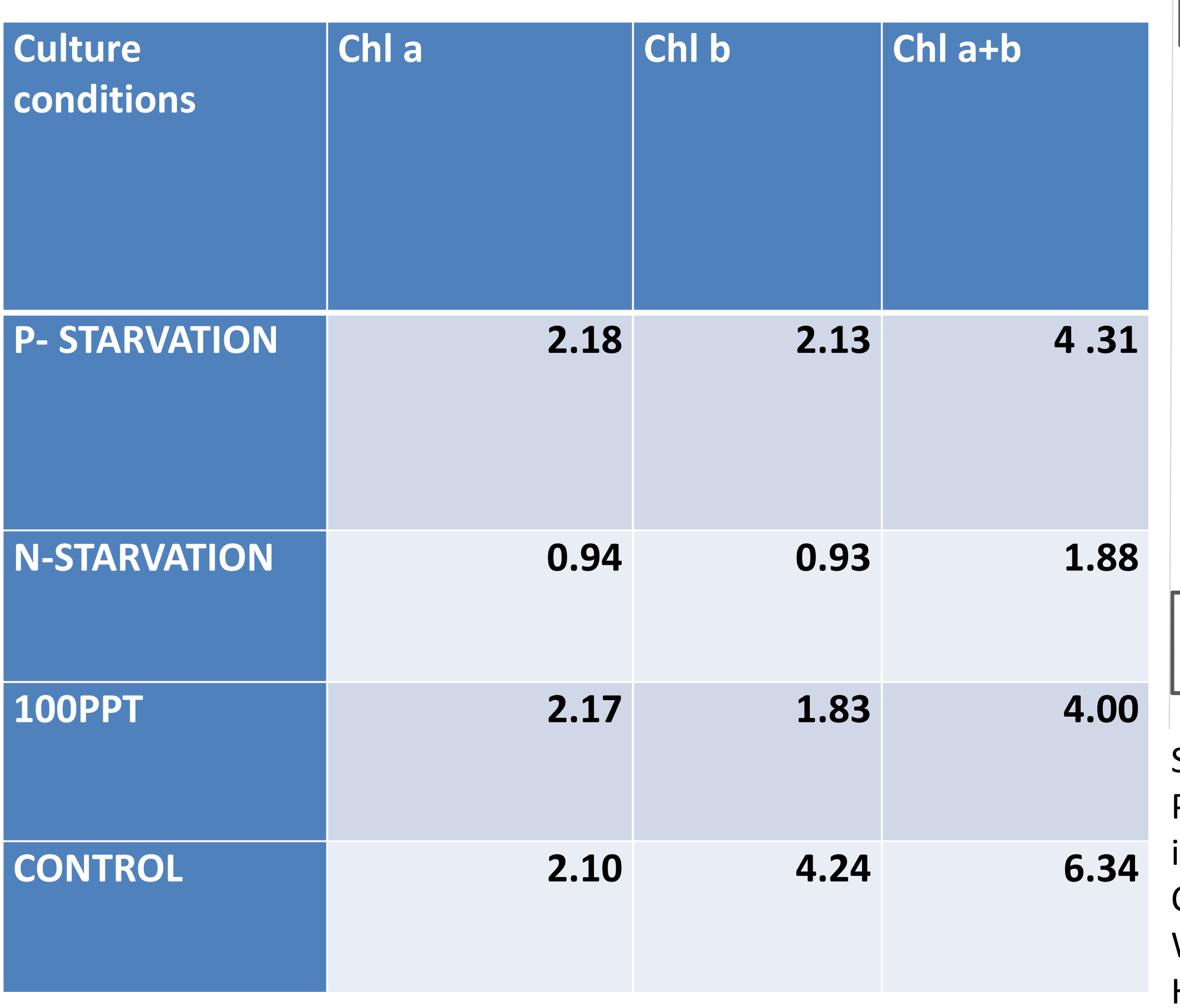

The analysis of the effect of the different regimes applied on the production of photosynthetic pigments showed a dramatic decrease $(\mathbf{7 0 . 3 5 \%})$ of the total Chlorophyll ( $\mathrm{Chl} \mathrm{a}+\mathrm{b})$ in the case of N-Starvation followed by a decrease of $\mathbf{3 4}$ and $\mathbf{3 7 \%}$ in the case of PStarvation and $100 \mathrm{ppt}$ salinity. The drastic reduction in the Chlorophyll content observed was due to nitro deficiency.
RESULTS \&

\section{DISCUSSION}

3-Effect of culture conditions on lipid quantity and quality:

$\square 50$-control $\square 50-\mathrm{P} \quad \square 50-\mathrm{N}$ $\square 50-100 p p t \quad \square 50-15 \circ C$

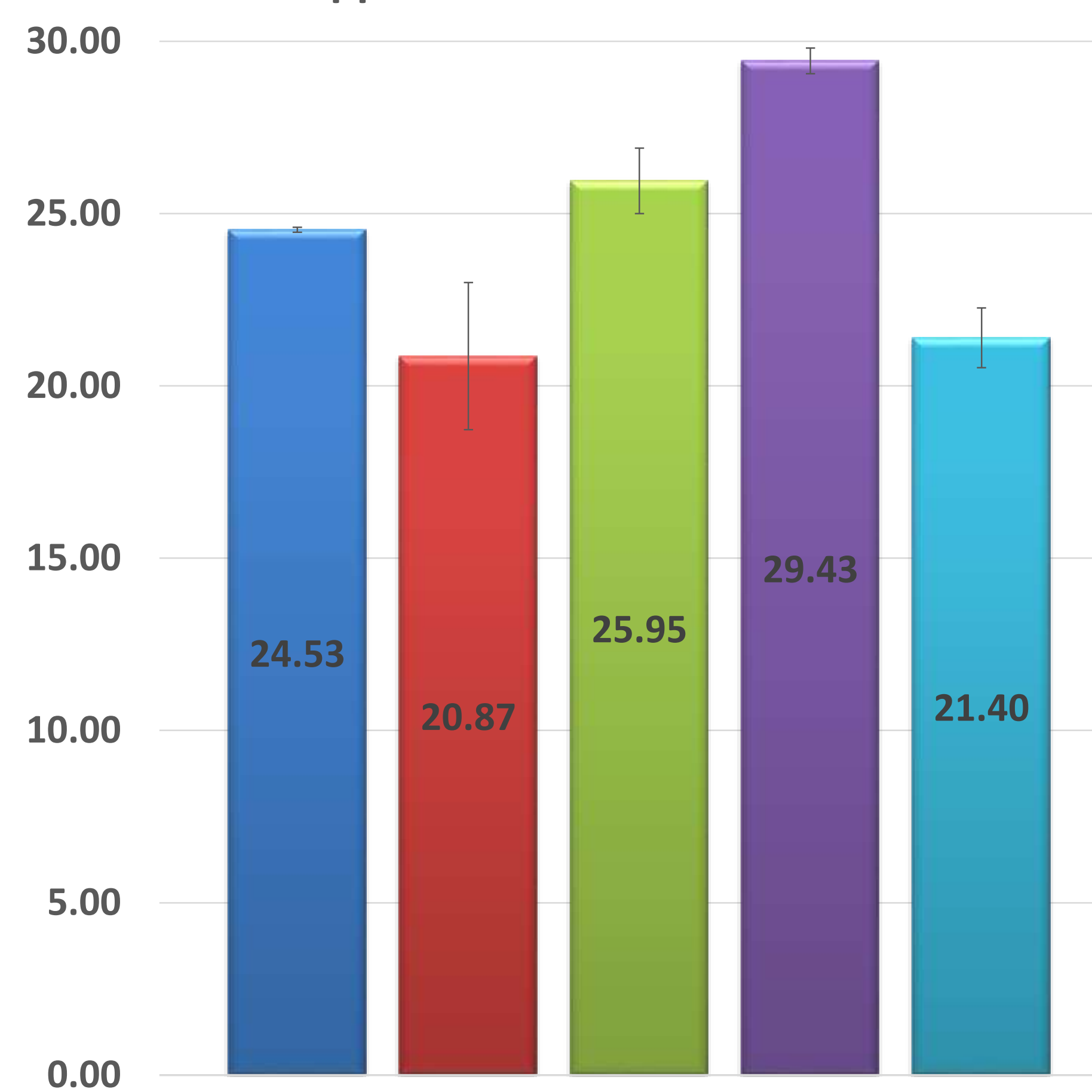

The comparison of the QUCCCM50 growth under the different cultivation regimes with the control showed that the 100ppt enhanced the lipid accumulation comparing to the control followed by $-\mathrm{N}$.

Table 3 . FAMEs profile under different culture condition

\begin{tabular}{|l|r|r|l|l|l|l|l|l|}
\hline $\begin{array}{l}\text { lipid } \\
\text { name }\end{array}$ & Control & & & & & & & \\
\hline
\end{tabular}
an increase in the ALA concentration in the case of $N$ starvation and an appearance of DHA in the case of high salinity regime. Such result proved that we can modulate the FAME production via dealing with the cultivation conditions to optimize the quantity and quality of algal fatty acids.

\section{CONCLUSION}

The physicochemical conditions of growth when optimized led to the improvement of our strain QUCCCM 50 in terms of metabolite production. Different stress conditions exhibited difference in lipid quantity and quality. The best condition found was using media with 100ppt salinity, which led to an increase of $25 \%$ of lipid quantity and had a major effect on the lipid profiling making it suitable to enhance the feed quality.

\section{REFERENCES}

aadaoui, I., Al Ghazal, G., Bounnit, T., Al Khulaifi, F., Al Jabri, H., \& potts, M. (2016). Evidence of thermo and halotolerant Nannochloris solate suitable for biodiesel production in Qatar Culture Collection of (1) Wells, M. L., Potin, P., Craigie, J. S., Raven, J. A., Merchant, S. S., Helliwell, K. E.,... \& Brawley, S. H. (2017). Algae as nutritional and applied phycology, 29(2), $949-982$.

Zhu, L. D., Li, Z. H., \& Hiltunen, E. (2016). Strategies for lipid production improvement in microational, 2016.

\section{Acknowldgement}

